\title{
Defining Priorities to Improve Patient Experience in Non-Muscle Invasive Bladder Cancer
}

\author{
Tullika Garg ${ }^{\mathrm{a}, \mathrm{b}, *}$, Jill Nault Connors ${ }^{\mathrm{c}}$, Ilene G. Ladd ${ }^{\mathrm{b}}$, Tyler L. Bogaczyk ${ }^{\mathrm{b}}$ and Sharon L. Larson ${ }^{\mathrm{b}}$ \\ ${ }^{a}$ Department of Urology, Geisinger, Danville, PA, USA \\ ${ }^{\mathrm{b}}$ Department of Epidemiology and Health Services Research, Geisinger, Danville, PA, USA \\ ${ }^{\mathrm{c}}$ Department of Emergency Medicine, Indiana University School of Medicine, Indianapolis, IN, USA
}

\begin{abstract}
.
Background: Although approximately $75 \%$ of bladder cancers are non-muscle invasive (NMIBC) at diagnosis, most research tends to focus on invasive disease (e.g., experiences related to radical cystectomy and urinary diversion). There is a lack of studies on quality of life, and especially qualitative research, in bladder cancer generally. As a result, relatively little is known about the experiences and needs of NMIBC patients.

Objective: To understand patient experience, define care priorities, and identify targets for care improvement in NMIBC across the cancer continuum.

Methods: Through focus groups, patients treated for NMIBC (stage $<\mathrm{T} 2$ ) were invited to share their care experiences including diagnosis, treatment, and survivorship. Transcripts were analyzed using conventional content analysis to identify themes and subthemes.

Results: Twenty patients (16 male, 4 female, all white) participated in three focus groups. Five primary themes emerged: access to care, provider characteristics and communication, quality of life, goals of care/influences on decision-making, and role of social support. Patients with NMIBC desired timely access to care and honest and caring provider communication. They described urinary function and emotional quality of life changes resulting from diagnosis and treatment. Avoiding cystectomy and being alive for family were the major decision influencers.

Conclusion: In this qualitative study, we identified access to care, provider characteristics and communication, quality of life, values/influences on decision-making, and social support as priority areas to improve patient experience in NMIBC. Care redesign efforts should focus on improving access, enhancing provider communication, reducing side effects, and supporting caregiver roles.
\end{abstract}

Keywords: Urinary bladder neoplasms, qualitative research, patient-centered care, urologic neoplasms

ABBREVIATIONS

BCG

GHS

NICE bacillus Calmette-Guerin Geisinger Health System National Institute for Health and Care Excellence

\footnotetext{
*Correspondence to: Tullika Garg, MD, MPH, Clinical Investigator, Department of Urology, Geisinger, 100N. Academy Ave., Danville, PA 17822, USA. Tel.: +1 570271 6328; Fax: +1 570217 6955; E-mail: tgarg@geisinger.edu.
}

$\begin{array}{ll}\text { NMIBC } & \text { Non-muscle invasive bladder cancer } \\ \text { TURBT } & \text { Transurethral resection of bladder tumor } \\ \text { UK } & \text { United Kingdom }\end{array}$

\section{INTRODUCTION}

Bladder cancer is the fifth most commonly diagnosed cancer in the United States with over 79,000 new cases diagnosed annually [1]. Most of these (75-80\%) are non-muscle invasive at diagnosis (stage 
$<\mathrm{T} 2$ ) and have relatively low risk of progression to invasive or metastatic disease (10-20\%) [2]. However, the risk of recurrence is high ranging from $30-70 \%[3,4]$, and there are currently over 600,000 bladder cancer survivors in the United States [5]. Despite the relative rarity of muscle-invasive bladder cancer, much of current research is directed toward the treatment of patients undergoing major surgery, such as radical cystectomy and urinary diversion [6]. As a result, significant gaps remain in our understanding of the needs and overall patient experience in non-muscle invasive bladder cancer (NMIBC) across the cancer continuum.

Patient experience is increasingly being recognized as an important metric since its inclusion as one of the "Triple Aims" for the U.S. health care system [7]. Poor care satisfaction and experiences have been associated with outcomes such as lower health-related quality of life, mental well-being, and decreased adherence to care [8-10].

A survey comparing the treatment experiences of all-cause cancer among over 40,000 cancer patients found that bladder cancer patients have among the lowest levels of satisfaction with care when compared to other urologic cancer sites and cancer generally [11]. Much of current research has focused on quantifying sexual function and health-related quality of life, which are not fully inclusive of a patient's experience through the cancer continuum. Understanding other aspects of patient experience and care such as goals of treatment, treatment burden, care processes, financial toxicity, and caregiver concerns are needed to redesign care with a patient focus.

Due to the association between patient experience and outcomes, engaging patients to understand care processes and experiences across the cancer continuum has been defined as a priority area for multiple professional organizations and advocacy networks, particularly in bladder cancer [12, 13]. Qualitative research methods offer the advantage of directly engaging patients to share their perspective; however, exceedingly few qualitative studies pertaining to NMIBC exist. A recent systematic review identified fourteen qualitative studies of patient experience in bladder cancer, and only two were specific to NMIBC [14].

We designed a qualitative focus group study of adults with NMIBC to understand patient experience and define care priorities. We sought to understand processes across the cancer continuum including diagnosis, decision-making, treatment, and survivorship.

\section{MATERIALS \& METHODS}

\section{Study design}

In this qualitative study, we conducted three focus groups with patients from the Geisinger Health System (GHS). The study was reviewed by Geisinger's Institutional Review Board and deemed exempt due to minimal risk.

\section{Setting and participants}

Eligible participants were adults 18 years or older and diagnosed with NMIBC (stage <T2) between January 2000 and May 2016. Participants were identified via the GHS Cancer Registry and by screening urologist clinics. From this list, a random group of eligible participants received a mailed recruitment letter and a follow-up phone call from a study team member. Recruitment was performed from August through October of 2016. As this was a pilot study with budget limitations, participants were recruited until all focus group slots were filled.

A total of 87 patients were approached and 20 participated in the focus groups. Participants ranged in age from 46 to 85 years, were $80 \%$ male, and were $100 \%$ white.

\section{Focus group guide}

A semi-structured focus group guide was created to explore patient experience. Participants were asked to describe their cancer story starting with how they found out they had bladder cancer. Subsequent questions touched on decision-making, goals of care, and how life has changed since diagnosis (Supplement).

\section{Data Collection}

All three focus groups were conducted in November, 2016, with an average attendance of 7 participants per group. Two focus groups took place in the evening and one was held in the afternoon. Groups were conducted in English and lasted 90 minutes. Participants received a catered meal and a $\$ 25$ gift card. Each session was audio recorded and transcribed verbatim by a study team member. All identifying information was removed from the transcripts to maintain anonymity. All authors except T.G. assisted in leading the focus groups. T.G. is a urologic oncologist and multiple participants were actively receiving treatment in her practice. She did not lead 
any sessions in order to allow participants to speak freely about their experiences.

\section{Data analysis}

Transcript coding occurred from January through March 2017 during monthly research team meetings. As little is known about the NMIBC patient experience, we selected conventional content analysis to code the transcripts [15]. This type of analysis is used to describe a phenomenon that has not been described before through the process of data immersion. Four researchers (T.G., J.N.C., I.G.L., and T.L.B.) under the direction of a qualitative research expert (S.L.L.) read the transcripts carefully multiple times, iteratively identified keywords and phrases, and developed a limited preliminary code list. The study team then discussed the preliminary list and developed a mutually agreed upon codebook of overarching themes. Researchers then read each transcript again and performed line-by-line coding. Disagreements were discussed and resolved when possible. There was high concordance between coders' assessments of key themes and phrases and the assignment of content to categories. Consensus between focus groups was reached early in the process.

\section{RESULTS}

Five primary themes emerged from analysis including access to care, importance of provider characteristics and communication, quality of life outcomes, goals of care/influences on decision-making, and role of social support (Table 1).

\section{Access to care}

Focus group participants expressed concerns about gaining access to urologic care, both in terms of care providers and treatment options. They noted that it seemed difficult to recruit urologists. More specifically, participants expressed concerns about lack of continuity of care due to physician turnover. They described difficulty obtaining timely appointments in the urology department after referral, and worries about the impact of delayed care on cancer progression. Participants wanted to have evaluation and treatment close to home rather than traveling long distances to major cities. Some individuals had experienced the 2014 bacillus Calmette-Guerin (BCG) shortage. They described receiving alternate medications or reduced dosages of BCG which again led to worry and stress. In relation to this, some were worried about future access to standard of care treatments for NMIBC due to national and international drug shortages.

However, while most participants described access barriers, one participant discussed how a single contact person such as a nurse or patient navigator in the urology department facilitated access.

\section{Provider characteristics and communication}

Focus group participants shared both positive and negative experiences interacting with healthcare providers such as urologists, advanced practitioners, and nursing staff. Most spoke very highly of interactions with all healthcare providers; however, some described poor experiences related to physician communication of the diagnosis. For example, one participant received his/her bladder cancer diagnosis via secure email messaging from a physician who was not one of his/her primary providers.

The groups felt that there was room for improvement with the surveillance cystoscopy visits and that the format of the visit did not leave time to discuss the findings or other aspects of survivorship care such as diet and lifestyle. Participants also expressed a desire for prompt post-surgical communication with providers after ambulatory surgery for transurethral resection of bladder tumor (TURBT). They felt that the time period between surgery and the visit to discuss the pathology report was too long adding unnecessarily to anxiety about results.

Participants described characteristics of high quality communication and praised providers who gave thorough explanations about diagnostic tests and treatments. Many participants wanted their providers to render the diagnosis honestly and directly. They felt that they needed to have confidence in the urology provider and the healthcare system. Participants were very appreciative of the hard work of nurses and after-hours communications of physicians and described how these actions made them feel special and cared for.

\section{Quality of life}

One of the most significant themes to emerge was how NMIBC treatments affected people's lives both physically and emotionally. A primary complaint was changes in voiding habits due to the multiple TURBTs and intravesical treatments. All three focus groups reported frequency, urgency, and nocturia leading to 
Table 1

Themes and subthemes with representative quotations

Themes and Subthemes
1. Access to Care: Facilitators, Barriers, and Preferences
Barriers
-BCG Shortage
-Urologist Shortage

Representative Quotations

- "she couldn't get the [BCG] she wanted so she gave me 6 weeks of treatment of some other medicine"

- "for me to wait a whole year for a doctor because they are so short of doctors in this hospital and this is their complaint all the time they don't have enough doctors to take care of the patients. And I think the patient/doctor relationship is really going down the drain because they are so busy and they can't handle the patients."

Preferences

- "I wanted to be here at Geisinger rather than hauling to Maryland or Philadelphia or someplace like that."

Facilitators of access

- "A nurse essentially is what she is but she is sort of a... I don't know if I am on her caseload or whatever but she is my 1 contact at urology whenever I am about to have a procedure or right after I have a procedure. If I have questions or anything, I have always felt like there was a person there other than a doctor, because you know how hard it is to get a doctor on the phone."

2. Provider Characteristics and Communication Good explanations of conditions

Honesty about diagnosis and treatment

Caring

-After-hours communication

-Appreciation for allied health \& nursing staff

Importance of second opinions

Need for confidence in provider

Need for prompt post-surgical communication

Poor interactions with providers

3. Impacts of Diagnosis and Treatment on Quality of Life

Concerns about hematuria

Difficulty with catheters
- "And when I would go in for the scope every 3 months he would flip the camera around and say now here is what I did here, here is what I did there and I'd say I don't think anybody could have explained it any better than he did."

- "And he certainly wasn't my friend. Very blunt. Very honest. Very truthful and didn't really sugar coat anything so... which personally I would rather have it that way."

- "You know the calling at home. I had calls from Dr. X at home in the evening or on the weekend and that is really important. I think it tells you that they really do care and I know that probably all of us being the age we are that we recall the family doctor that came to the home and made home visits. You don't have that today and it is an inconvenience in medical care today I think in some respects and I think that the phone call really means a lot."

- "Well you have to give those poor nurses credit because those poor things just run from one room to another. They do their job but they are on the go. I just spent last Thursday in the hospital and I just got a card recently wishing me well and there were 16 nurses that signed it."

- "I think that second opinions are good. I think it allows the patient to really realize and get a broader view of your condition and then the treatment for it and so forth."

- "Attitude of the patient, confidence in the doctor and the entire Geisinger staff. You have to have the confidence in there because that is a big thing to getting better."

- "I would have liked to have had a follow-up after the surgery because right after the surgery you can tell me anything you want and I'm not going to remember it. . . so I think a follow-up call of some kind, either from the doctor or from another knowledgeable person about me, because I would have had some questions, "what did you find?", "how bad was it?"

- "I was very angry when I found out [about the cancer] because I found out via email from a doctor who wasn't even mine."

- "It is kind of weird to go either once a year or every 6 months and all the doctor does is insert a scope in you and look around and say "oh I don't see anything" or "oh I see something" and then see you in another 6 months. To me it's not a classic doctor/patient relationship. It's sort of like there is no afterward discussion about "here is what I saw" and then "what have you been doing?"

- "I had 15 treatments of BCG and he said [the cancer] was clear. Every time I got a treatment, I would hemorrhage bad."

- "I had to wear a big pair of baggy pants to my grandson's graduation because there was a bag there and I am feeling it every couple minutes to see if I have to go to empty it. That was just hard..." 
Table 1

(Continued)

\begin{tabular}{ll}
\hline Themes and Subthemes & Representative Quotations \\
\hline Changes in urinary function & "I can't go on a trip or anything. I have to know where the bathrooms are all the \\
time. Like I went down to see my daughter in Jersey last week. I stopped 6 times on \\
the way down. Finally, you know what I did? I stopped at McDonalds and picked up \\
a cup and believe me I used it. I had to, but you know you have to plan everything. I \\
can't go anyplace without planning. I've got to make sure I have a bathroom." \\
- "the only thing that changed is that before I had the bladder cancer and the surgery \\
and the treatments, I could go to sleep at night and get up the next morning and go to \\
the bathroom. Now it's 2 or 3 times a night." \\
- "The cystoscopy was nothing. The after surgery stuff was brutal." \\
- "That was the first major surgery that I have ever had. Ever since the surgery, I feel a \\
little differently. Like not my normal self. Kind of walking around in a dizzy state \\
for a little while." \\
- "And I had all sorts of fear. I never expected cancer." \\
" "Yeah I don't have fear either. I just go for my treatments and I just live like I don't \\
host-surgery side effects \\
- "And I know nobody has really talked about this but when it has to do with your \\
penis and that whole area... I don't know male or female it probably isn't \\
Fear, anxiety, and embarrassment \\
something you really want to share with anybody about when there is something \\
wrong down there."
\end{tabular}

4. Goals of Care and Influences on Treatment Decisions

Avoiding cystectomy

- "Well, I didn't want my bladder out. I decided I was not going to remove the bladder. I had the idea that I would rather live with a bladder in and die sooner."

- "We will go through almost anything instead of removal of the bladder from the body. We lose sexual performance, that's gone. And then the bag outside, which is... we had the catheter with the bag and that was bad enough but to have it forever, that is a situation that we have to look at. If we lose the bladder then we have to carry the bag forever so we don't want to lose that under any circumstances."

- "What kind of bummed me out a little bit is how I have had the wash twice and each time it was like they wanted to push to remove everything. I would say if my lifestyle would allow it I probably would have went for it but my lifestyle... my work. I really couldn't go for a bag."

Avoiding death

- "I keep going from year to year but I really didn't want surgery but I don't want cancer to take me either."

- "I can see the point of, "yeah, we want to save your life." We take everything out and you are good."

Being alive for family and other supporters

- "I would rather get [the bladder] out and give me the satisfaction of knowing that it is not there and not be scared and worried and crying every day because I have 12 granddaughters and 3 grandsons that I want to be with."

5. Role of Social Support

Spouse, family, friends

- "I'll do my cooking and baking, my husband does the house work, he does the laundry because I have trouble walking, especially in my spine area because of the spinal stenosis and [friend] visited me and never left. But my family, my youngest son and oldest son and husband I couldn't ask for more."

Other providers

Focus group

- "My own primary care physician, I speak to him about everything."

- "This is now my support group. This is the only one that I have been to. I mean I have learned some things tonight that when I go to urology now I will ask about."

disrupted sleep and fatigue. Individuals described challenges with travel or going out in public, needing to find the nearest bathroom, and transitioning to adult diapers to manage their urinary symptoms. Additional challenges following TURBT included pain and changes in cognitive function. Discomfort with postoperative catheters was universal. Hematuria was a distressing side effect of intravesical treatment, and in some instances, a complication after TURBT.

Emotional changes were noted beginning with the diagnosis. Common reactions included expressed embarrassment due to the close approximation of the bladder to genitalia, as well as fear and "devastation" when they found out they had cancer. Others were matter-of-fact, feeling that fear was counterproductive to getting through treatment. Several participants consciously transferred their fear to the healthcare providers, stating that it was the providers' job to worry about the cancer, not theirs. Some described how the diagnosis was something that could not be changed, thus they put their fears aside in order to focus on treatment. Levels of fear were related to age in 
that younger participants ( $<60$ years) described more fear and devastation than older participants who were grateful for each day and stoic regarding the diagnosis.

\section{Goals of care and influences on treatment} decisions

Treatment decisions, and the factors that had gone into them, were a major point of discussion, especially in relation to radical cystectomy. There was acknowledgement that radical cystectomy might relieve the constant uncertainty of NMIBC recurrence, but most sought to avoid radical cystectomy at all costs, even if it meant enduring the side effects of multiple TURBTs and intravesical therapy. One participant relayed the experience of someone in his/her community who committed suicide after radical cystectomy due to poor quality of life. Another felt that having an ostomy would interfere with his/her livelihood. Two participants expressed a willingness to undergo radical cystectomy in order to prolong life and be alive for family.

\section{Role of social support}

Support systems are critical to managing cancer diagnosis and treatment. Spouses were identified as the primary social support in all three focus groups. Spouses helped participants quit smoking, make dietary changes, and incorporate daily exercise. They also kept track of appointments and medications, and helped with household duties. Other supports, such as grandchildren and friends provided comfort. Additionally, adult children with medical or science backgrounds were a resource for information gathering about their condition.

Primary care and alternative medicine providers were identified in all three groups as important supports. A few participants had attended diseasespecific support groups and found them to be useful for information gathering and obtaining support from other patients. Those who had never attended a support group found participating in the focus group to be a positive experience and in that being in this focus group study for 1-2 hours was a support in itself.

\section{DISCUSSION}

Much of the current qualitative research in bladder cancer is focused on the impact of major extirpative surgery for muscle invasive disease. However, the majority of patients have NMIBC at diagnosis and rarely progress to invasive disease. NMIBC has its own set of challenges for patients including a high recurrence rate, uncomfortable instrumentation of the urinary tract for disease surveillance, frequent outpatient surgeries, and treatment with intravesical therapies. The effects of these challenges were captured via the experiences reported in this qualitative focus group study of NMIBC patients. We identified five common themes across the cancer continuum including access to care, provider characteristics and communication, quality of life, influences on treatment decision-making/goals of care, and role of social support.

Being treated for cancer is not a positive experience, but international studies suggest that the patient experience for bladder cancer is especially poor. The United Kingdom (UK) National Institute for Health and Care Excellence (NICE) guidelines for bladder cancer highlighted the phenomenon of low satisfaction in this population [16]. The 2013 Cancer Patient Experience Survey of 66,418 cancer patients from the United Kingdom found that urologic cancer patients (excluding prostate) scored lowest on twelve indicators such as written assessments and care plans, information about support groups, and contact information for a clinical nurse specialist [16]. Bladder cancer patients had the poorest satisfaction level with treatment decision-making, resulting in a call to pursue qualitative research to determine and address causes [11].

Despite this, few studies have explored the causes of poor patient experience in bladder cancer, especially in NMIBC. Our findings both corroborate and expand upon limited existing qualitative studies. The study by Rutherford, et al. described how side effects such as urgency, frequency, nocturia, hematuria, and pain with urination influenced dayto-day living such as social participation and travel, cognitive function, and emotional well-being. The importance of clear provider communication, support from the healthcare system, and continuity of care were also identified [17]. A recent mixed-methods study with focus groups and surveys examining attitudes towards surveillance cystoscopy in twelve NMIBC patients in the Veterans Affairs system found that NMIBC patients experienced anxiety around the time of surveillance cystoscopy and have worry about disease progression and metastasis. These patients, similar to our study, had a wide range of perceptions about their level of involvement with decisions about surveillance from deferring to the physician to high levels of involvement [18]. 
Physician communication and decision-making style is associated with a variety of outcomes in cancer patients including trust and participation, selfefficacy, perceived control, uncertainty, and mental health-related quality of life [19]. A qualitative study of bladder cancer patients from major cancer centers described the importance of clear, compassionate, and honest communication about treatment options, side effects, and prognosis [20]. Another qualitative study described how urologic cancer patients wanted to be treated in a caring manner, as someone who matters [21]. Similarly, our participants' valued provider accessibility and caring attitudes. Teaching effective and caring provider communication may be an important target to improve patient experience, and is one recommendation that has been implemented systemwide at our institution and others through the C.I. C.A.R.E model [22, 23].

A difference between our study and previous studies is the degree to which participants discussed access to care. This likely reflects our rural setting. While we are facing a nationwide urologist shortage, rural areas are particularly hard-hit due to retirement of older urologists and the preference of younger urologists to practice in urban areas [24]. In addition to concern about the potential downstream effects of diagnosis and treatment delays, our participants desired care continuity and suggested having a single point of contact or a navigator embedded within the urology clinic. The UK NICE review found that this intervention was associated with improved patient satisfaction [16]. In addition to improving patient satisfaction, patient navigator programs have been shown to reduce health care utilization and costs. In a study from the Centers for Medicare and Medicaid (CMS), a patient navigator program was associated with fewer hospitalizations and emergency department visits [25].

The patient perspective of the 2014 BCG shortage was a timely finding, but oncology drug shortages have been increasing [26]. To our knowledge, ours is the only study to touch on the patient perspective of the BCG shortage, demonstrating both the importance of steady access to lifesaving drugs and uncertainty that can arise from intermittent access.

Social support was a significant theme in our study and is associated with health-related quality of life among cancer survivors [27]. Cancer survivors lacking a partner are more likely to report poor physical and mental quality of life [28]. The previously described qualitative study of bladder cancer patients from major cancer centers identified "family issues" as a prominent theme in treatment decisionmaking [20]. Family members had roles similar to those elucidated in our study, including information gathering and attendance at clinical encounters. Our study highlights the fact that patients recognize social support as an integral part of a successful journey through the cancer continuum. Providers should consider the social context around an individual patient, and, when possible, seek partnerships with informal caregivers to try to improve patient experience.

The present study represents the first study to evaluate patient experience in NMIBC using qualitative methods; however, our findings must be interpreted within the context of certain limitations. We conducted a limited number of focus groups with a convenience sample of patients. It is possible we did not reach thematic saturation and that with more focus groups, we may have identified additional themes. This study was conducted in a single, rural health system and though most themes were similar to what has previously been reported in the literature, we did identify a few themes that may not be applicable to patients in other health systems or in urban settings. All of our participants were white, reflecting the race/ethnicity of the GHS catchment area, and thus the findings may not generalize to more diverse populations.

This strengths of this study include its range of ages and the enthusiastic engagement on the part of participants. Previous studies were performed in urban, academic medical centers. Our study, while it identified universal themes, also highlights the unique perspectives of rural patients in community-based healthcare systems. Finally, this is one of the few existing studies that applies a qualitative approach to understanding patient priorities and experience in bladder cancer, and the largest focus group study conducted in NMIBC [18].

\section{CONCLUSIONS}

In this qualitative study, we identified access to care, provider characteristics and communication, quality of life, values/influences on decision-making, and social support as priority areas to improve patient experience in NMIBC. Care redesign efforts should focus on improving access, patient navigation and care coordination, teaching providers caring communication, reducing side effects and emotional impacts, and supporting the roles of informal caregivers. 


\section{ACKNOWLEDGMENTS}

The authors would like to acknowledge the patients who shared their time and stories with us. This research was supported by a grant from the Geisinger Clinic Research Fund (SRC-S-64).

\section{CONFLICT OF INTEREST}

The authors have no conflict of interest to report.

\section{FUNDING}

This research was supported by a grant from the Geisinger Clinic Research Fund (SRC-S-64).

\section{CONFLICTS OF INTEREST}

The authors have no conflict of interest to report.

\section{REFERENCES}

[1] Siegel RL, Miller KD, Jemal A. Cancer statistics, 2017. CA Cancer J Clin 2017;67:7-30.

[2] Nargund VH, Tanabalan CK, Kabir MN. Management of non-muscle-invasive (superficial) bladder cancer. Seminars in Oncology 2012;39:559-72.

[3] Nielsen ME, Smith AB, Meyer A-M, et al. Trends in stage-specific incidence rates for urothelial carcinoma of the bladder in the United States: 1988 to 2006. Cancer 2013;120:86-95.

[4] Chang SS, Boorjian SA, Chou R, et al. Diagnosis and treatment of non-muscle invasive bladder cancer: AUA/SUO guideline. J Urol 2016;196:1021-9.

[5] SEER Cancer Stat Facts [Internet][cited 2017 Dec 27] Available from: https://seer.cancer.gov/statfacts/html/urinb.html

[6] Yang LS, Shan BL, Shan LL, et al. A systematic review and meta-analysis of quality of life outcomes after radical cystectomy for bladder cancer. Surg Oncol 2016;25:281-97.

[7] Berwick DM, Nolan TW, Whittington J. The triple aim: Care, health, and cost. Health Affairs 2008;27:759-69.

[8] Barbosa CD, Balp M-M, Kulich K, et al. A literature review to explore the link between treatment satisfaction and adherence, compliance, and persistence. PPA 2012;6:39-48.

[9] Crow R, Gage H, Hampson S, et al. The measurement of satisfaction with healthcare: Implications for practice from a systematic review of the literature. Health Technol Assess 2002;6:1-244

[10] Bjertnaes OA, Sjetne IS, Iversen HH. Overall patient satisfaction with hospitals: Effects of patient-reported experiences and fulfilment of expectations. BMJ Qual Saf 2012;21:39-46.

[11] Turabi El A, Abel GA, Roland M, et al. Variation in reported experience of involvement in cancer treatment decision making: Evidence from the National Cancer Patient Experience Survey. British Journal of Cancer 2013;109:780-7.
[12] Kent EE, Mitchell SA, Castro KM, et al. Cancer care delivery research: Building the evidence base to support practice change in community oncology. J Clin Oncol 2015;33: 2705-11.

[13] Filippou P, Smith AB. Prioritizing the patient voice in the development of urologic oncology research. Urol Oncol 2017;35:548-51.

[14] Edmondson AJ, Birtwistle JC, Catto JWF, et al. The patients' experience of a bladder cancer diagnosis: A systematic review of the qualitative evidence 2017:1-9.

[15] Hsieh H-F, Shannon SE. Three approaches to qualitative content analysis. Qualitative Health Research 2005; 15:1277-88.

[16] UK NICE Guideline. Bladder Cancer: Diagnosis and Management [Internet][cited 2017 Dec 29] Available from: https://www.nice.org.uk/guidance/ng2

[17] Rutherford C, Costa DSJ, King MT, et al. A conceptual framework for patient-reported outcomes in non-muscle invasive bladder cancer 2017:1-8

[18] Koo K, Zubkoff L, Sirovich BE, et al. The burden of cystoscopic bladder cancer surveillance: Anxiety, discomfort, and patient preferences for decision making. URL 2017;108:122-8.

[19] Arora NK, Weaver KE, Clayman ML, et al. Physicians? decision-making style and psychosocial outcomes among cancer survivors. Patient Education and Counseling 2009;77:404-12.

[20] Berry DL, Nayak M, Halpenny B, et al. Treatment Decision Making in Patients with Bladder Cancer. BLC 2016;1: 151-8.

[21] Skea ZC, MacLennan SJ, Entwistle VA, et al. Communicating good care: A qualitative study of what people with urological cancer value in interactions with health care providers. European Journal of Oncology Nursing 2014;18:35-40.

[22] Astonishingly Effective Ways This Healthcare Leader is Reinventing The Hospital Experience. [Internet][cited 2017 Dec 29] Available from: https://www.forbes.com/sites/car minegallo/2016/09/29/5-astonishingly-effective-ways-this -healthcare-leader-is-reinventing-the-hospital-experience/ $\# 132170597 \mathrm{a} 2 \mathrm{~b}$

[23] C.I.C.A.R.E. UCLA Health [Internet][cited 2017 Dec 29] Available from: https://www.uclahealth.org/patientexperience/cicare

[24] Gaither TW, Awad MA, Fang R, et al. The near-future impact of retirement on the urologic workforce: Results from the american urological association census. Urology 2016;94:85-9.

[25] Colligan EM, Ewald E, Keating NL, et al. Two innovative cancer care programs have potential to reduce utilization and spending. Med Care 2017;55:873-8.

[26] Kehl KL, Gray SW, Kim B, et al. Oncologists' experiences with drug shortages. Journal of Oncology Practice 2015;11:e154-62.

[27] Westby RP, Berg CJ, Leach C. Gender, race, BMI, and social support in relation to the health-related quality of life of cancer survivors: A report from the American Cancer Society's Study of Cancer Survivors II (SCS-II). Quality of Life Research 2015;25:409-21.

[28] Jensen RE, Arora NK, Bellizzi KM, et al. Health-related quality of life among survivors of aggressive non-Hodgkin lymphoma. Cancer 2013;119:672-80. 